\title{
APLICAÇÃO DO TESTE DE ELEGIBILIDADE MULTIDIMENSIONAL NA DEFINIÇÃO DO PÚBLICO-ALVO BENEFICIÁRIO DE POLÍTICAS PÚBLICAS*
}

\author{
Ana Lucia Cosenza Faria ${ }^{* *}$ \\ Denise Britz do Nascimento Silva ${ }^{* * *}$ \\ Carmem Aparecida Feijó ${ }^{* * * *}$
}

RESUMO Este artigo apresenta o Teste de Elegibilidade Multidimensional como um método alternativo de seleção de beneficiários para políticas sociais no caso brasileiro. Como a aplicação do referido método requer a utilização de modelos estatísticos com dados coletados em pesquisas domiciliares por amostragem, elabora-se um modelo de regressão logística que relaciona a probabilidade de um domicílio estar em condição de pobreza com suas características físicas e com variáveis socioeconômicas de seus moradores. As probabilidades previstas pelo modelo constituem os escores de propensão (propensity scores) à pobreza que podem ser utilizados como critério de inclusão de domicílios em programas de transferência de renda. As estimativas dos escores de propensão e uma medida de desempenho da focalização foram calculadas com base nas informações da PNAD 2003.

Palavras-chave: focalização de políticas públicas; teste de elegibilidade multidimensional; indicador de focalização

Código JEL: I 32

\footnotetext{
* Artigo recebido em 12 de abril de 2006 e aprovado em 28 de maio de 2007.

** Mestre pela ENCE/IBGE. Tecnologista do CASNAV, e-mail: cosenzaana@ig.com.br

*** PhD pela Universidade de Southampton. Pesquisadora IBGE e professora da ENCE/IBGE, e-mail: denisesilva@ibge.gov.br

*** PhD pela University College London. Professora da UFF e Pesquisadora CNPq, e-mail: cfeijó@ terra.com.br
} 


\section{AN APPLICATION OF THE PROXY MEANS TEST TO DEFINE \\ THE TARGET POPULATION FOR PUBLIC BENEFITS}

ABSTRACT This paper presents the Proxy Means Test as an alternative method of selection of the target population to social policies in Brazil. The application of this method requires the employment of statistical methods on primary data collected by sampling household surveys. Based on PNAD 2003 we built a logistic regression model relating the probability of a household to be poor with its physical characteristics and other socioeconomic variables concerning the residents. The probabilities estimated by the model are the propensity scores to poverty and can be used as a norm to include the household in social programs. The estimates of the propensity scores and a measure of efficiency in the target population has been calculated with data from PNAD 2003.

Key words: target population and public policy; proxy means test; target population indicator 


\section{INTRODUÇÃO}

A tendência atual dos Governos no Brasil e no mundo de utilizar mecanismos de transferência de renda focalizados na população mais carente para combater a pobreza e a desigualdade ocorre junto com um amplo debate teórico sobre o tamanho do Estado na economia. Um desdobramento importante desse debate, e de interesse neste trabalho, é a discussão específica sobre os critérios para se identificar quais devem ser os beneficiários de políticas públicas de caráter social.

Desde 2001 o método utilizado no Brasil para determinar os beneficiários de programas de transferência de renda é o que a literatura denomina Teste de Renda Não Verificada, que é baseado na autodeclaração de renda das pessoas cadastradas no Cadastro Único (CadÚnico). Assim, os beneficiários são selecionados com base unicamente em sua renda, e como não há qualquer tipo de verificação da renda declarada, os indivíduos têm um incentivo a declarar rendas inferiores para tornarem-se beneficiários (De La Briere e Lindert, 2003). Esse método apresenta ainda outros problemas na coleta de dados sobre renda, particularmente em países com uma proporção muito grande de pessoas trabalhando no setor informal ou na agricultura, como é o caso do Brasil. Isso ocorre porque a renda proveniente de atividades dessa natureza pode variar muito durante um ano.

A partir de experiências com a utilização do Teste de Elegibilidade Multidimensional (conhecido na literatura como Proxy Means Test) na América Latina, observou-se que esse método representa uma alternativa viável para o atual sistema de seleção de beneficiários utilizado no Brasil. O procedimento consiste em utilizar dados de pesquisas domiciliares para descobrir quais são as variáveis úteis para explicar a renda (variável difícil de medir) e construir uma equação que discrimine os domicílios em condição de pobreza dos demais utilizando métodos estatísticos. Após a identificação da equação discriminante, aplica-se algum outro procedimento para que os potenciais beneficiários dos programas sociais se candidatem aos benefícios. Só então as características determinadas pelo método estatístico são investigadas empiricamente. Assim, a equação estatística funciona como uma forma de ordenar os domicílios de forma a priorizar o recebimento do benefício pela população em maior risco de pobreza.

Este artigo tem o objetivo de aplicar o Teste de Elegibilidade Multidimensional, a partir do ajuste de um modelo de regressão logística a dados 
coletados pela Pesquisa Nacional por Amostra de Domicílios 2003 (PNAD), para verificar a significância de um elenco de variáveis consideradas potenciais determinantes da probabilidade de um domicílio estar em condição de pobreza e obter uma estimativa do escore de propensão (propensity score) que discrimine a população investigada em dois grupos: pobres e não-pobres. Nosso exercício identificou 22\% de domicílios pobres na PNAD 2003, proporção consistente com as estimativas oficiais de domicílios pobres no País. Nosso exercício considerou também que o plano amostral utilizado na PNAD possui diversos aspectos que o diferenciam de uma amostra aleatória simples com reposição, tais como: estratificação, conglomeração e probabilidades desiguais de seleção. Portanto, as características do plano amostral utilizado na coleta de dados foram consideradas nas análises, utilizando o método da Máxima Pseudoverossimilhança.

Com a aplicação do método, foi possível selecionar os fatores e as ponderações dos fatores associados à condição de pobreza, utilizando os dados coletados pela PNAD 2003 e considerando em condição de pobreza os domićlios cuja renda domiciliar per capita era inferior a meio salário mínimo. Os resultados da aplicação do indicador de focalização apontaram para uma elevada proporção de domicílios pobres incluídos e baixa proporção de domicílios pobres excluídos, em todas as macrorregiões do País.

Este texto está dividido nas seguintes seções, além desta introdução: na seção 1 é apresentada uma breve referência ao debate recente sobre implementação de políticas sociais no Brasil; na seção 2 é apresentado o Teste de Elegibilidade Multidimensional (Proxy Means Test); na seção 3 é discutida uma medida de desempenho da focalização; na seção 4 é apresentada a aplicação do Teste de Elegibilidade Multidimensional; e, na seção 5 os resultados da Aplicação do Indicador de Focalização. Uma última seção apresenta nossas conclusões.

\section{DUAS POSIÇÕES SOBRE A IMPLEMENTAÇÃO DE POLÍTICAS SOCIAIS NO BRASIL}

O Brasil não pode ser considerado um país pobre, uma vez que se encontra entre as 10 maiores economias do mundo, segundo o Relatório de Desenvolvimento Humano de 2004. Essa constatação tem levado especialistas a rela- 
cionar a persistência dos altos índices de pobreza no Brasil à desigualdade de renda (Barros et al. 2001). No entanto, se há pontos de concordância na relação de causalidade entre desigualdade e pobreza no Brasil, há muitas discordâncias sobre a forma de como combatê-las e medi-las. Em 2003, dois estudos realizados por entidades públicas colocaram claro as divergências sobre a efetividade dos gastos sociais no Brasil. ${ }^{1}$ Em documento elaborado pelo Ministério da Fazenda, os autores apresentam uma avaliação do gasto social no Brasil enfatizando o fato de este não ser pequeno, mas mal distribuído. Um estudo da Prefeitura da cidade de São Paulo, por sua vez, diagnosticou que os gastos sociais focalizados não são suficientes para combater a desigualdade social.

O estudo do Ministério da Fazenda aponta que mais de dois terços das receitas líquidas do Governo Central Federal são utilizados para financiar despesas na área social. A maior parte desse gasto é destinada ao pagamento de aposentadorias e pensões que, segundo o documento, têm forte componente regressivo, em contraste com os gastos sociais com maior poder de enfrentamento da pobreza e das desigualdades sociais — tais como aqueles destinados à educação fundamental e média, à saúde e aos programas de renda mínima —, que, apesar de crescentes, ainda têm uma participação bastante reduzida no orçamento do Governo Federal. Os autores ressaltam a necessidade de realizar reformas que reduzam os privilégios concedidos pelo atual sistema, visando ao aumento da eficiência do gasto social, ${ }^{2}$ tais como a unificação dos programas de renda mínima e a necessidade de um sistema de monitoramento e avaliação de programas sociais. No que se refere aos programas de Garantia de Renda Mínima, o relatório destaca o fato de que, além dos programas federais, existem os programas municipais e estaduais, de forma que pode haver uma sobreposição, tanto de objetivos, quanto de público-alvo. Além disso, a multiplicidade de programas e de cadastros de beneficiários complica a gestão e o monitoramento dos diversos programas (Ministério da Fazenda, 2003).

No estudo realizado pela Prefeitura da Cidade de São Paulo, os autores apresentam outros dados e uma avaliação diferente do gasto social no Brasil, e argumentam que, apesar de o Brasil não ter construído um Estado de bem-estar social com características universais, houve, a partir da Constituição de 1988, algum progresso no sentido da universalização, questionado 
ultimamente. Para esses autores, a forma de romper com a desigualdade de renda e a pobreza no Brasil depende da construção de um sistema universal de políticas sociais com qualidade nos serviços prestados. O questionamento dos dados apresentados pelo Ministério da Fazenda se refere, principalmente, às escolhas metodológicas utilizadas para avaliar o gasto social (Prefeitura da Cidade de São Paulo, 2003).

Utilizando uma abordagem diferente da utilizada pelo Ministério da Fazenda, o trabalho apresenta uma interpretação contrastante: que os gastos sociais no Brasil são pequenos, em termos absolutos ou relativos, e que mesmo assim contribuem para atenuar a desigualdade de renda.

Quanto à argumentação do Ministério da Fazenda de que os destinatários dos gastos sociais no Brasil são os segmentos mais ricos da população, o segundo documento apresenta dois contra-exemplos: o seguro-desemprego, que nos anos 2001 e 2002 teve $80 \%$ dos benefícios concedidos a trabalhadores que recebiam no seu último trabalho menos que três salários mínimos; e o caso da Previdência Social, no qual o benefício médio no País era de cerca de dois salários mínimos.

Outra afirmação do estudo realizado pela Prefeitura da Cidade de São Paulo é que as aplicações financeiras e a estrutura tributária concentrada nos impostos indiretos são as responsáveis pelo aumento da distância entre os $10 \%$ mais ricos e os $10 \%$ mais pobres. Destaca ainda que as causas da desigualdade podem ser encontradas em três diferentes planos que se complementam: a dinâmica excludente do mercado de trabalho, a financeirização da economia e a estrutura tributária regressiva. Uma conclusão desse documento é que o aumento do gasto social e a implantação de impostos indiretos mais progressivos melhoram a distribuição de renda (Prefeitura da Cidade de São Paulo, 2003).

Salm (2003), debatendo os argumentos do documento do Ministério da Fazenda, considera que se trata basicamente de uma crítica à efetividade do gasto social brasileiro, pois, com a nossa elevada carga tributária, os impactos deveriam ser mais redistributivos do que os observados. $\mathrm{O}$ autor propõe uma reflexão conceitual sobre o gasto social. No que se refere à forma de distribuição dos gastos sociais, se pela via da focalização ou da universalização, argumenta que as transferências em dinheiro financiadas pelos impostos devem ser focalizadas nos mais pobres, mas a educação, saúde, previdência e segurança devem ser de acesso universal. 
Kerstenetzky (2005) aponta que a polarização do debate sobre a eficiência do gasto social no Brasil entre universalização e focalização deve ser ampliada para incluir a noção de justiça social. É o que veremos a seguir.

\subsection{Universalização versus focalização e a noção de justiça social}

Para Kerstenetzky (2005), a focalização per si não deve ser associada automaticamente à justiça social de caráter residual, nem tampouco a universalização à garantia de direitos sociais. São métodos alternativos e muitas vezes complementares de uma noção de justiça social que precisa ser previamente definida. A autora classifica a justiça social em justiça fina (ou de mercado) e justiça espessa.

Na justiça fina, uma economia de mercado seria dotada de uma capacidade integradora irresistível, pois seus benefícios materiais seriam transmitidos à quase totalidade da sociedade. Nesse caso, a política social seria reduzida à política econômica, com reformas de orientação mercadológica, que a longo prazo seriam capazes de incluir a todos.

Em uma concepção mais espessa, mesmo concedendo ao mercado a primazia na alocação de recursos econômicos, o Estado assumiria um papel maior na distribuição de vantagens socioeconômicas apoiado no argumento de que muitas desigualdades não se baseiam na escolha, ou na responsabilidade individual, mas em outras características, como a classe social, cor ou raça, gênero e até mesmo habilidades e talentos inatos.

A forte reação negativa de diversos intelectuais aos métodos de focalização reside na associação do método com o conceito de justiça fina, de caráter residual, na qual se acredita que o mercado seja capaz de se auto-ajustar. Entretanto, segundo Kerstenetzky (2005), a focalização pode ser utilizada como um instrumento, mesmo dentro de uma concepção mais espessa de justiça social, das seguintes maneiras:

(1) na busca do foco para solucionar um problema previamente especificado, em termos da eficiência do gasto social (ou seja, dada uma quantidade de recursos, determinar qual deveria ser a prioridade dos gastos, com base no conhecimento sobre a realidade demográfica, social e territorial do País); ou

(2) como ação reparatória para restituir a determinadas categorias direitos perdidos como resultado de injustiças passadas, o que implica 
que, sob esse aspecto, a focalização cumpriria o papel de complementar as políticas públicas universais.

Dessa forma, pode-se argumentar que, em uma sociedade desigual como o Brasil, algumas políticas focalizadas seriam importantes para a efetividade de direitos ditos universais, que, na prática, ainda encontram-se distantes da realidade do País. Os dois métodos - universalização e focalização — não são excludentes. É possível adotar um sistema de proteção social no qual haja a convivência de características do sistema residual e do sistema institucional.

Uma vez que a opção seja implementar programas sociais direcionados às pessoas de renda mais baixa, faz-se necessário utilizar algum método para definir o público-alvo beneficiário e examinar os possíveis custos e os benefícios envolvidos.

Um dos principais argumentos em favor da focalização nas políticas de combate à pobreza está relacionado à eficiência dos gastos sociais, ou seja, quanto mais preciso for o método utilizado para alcançar os pobres, menor será o desperdício e menores os custos envolvidos em alcançar o objetivo desejado. Uma forma simples de explicar a motivação principal para a utilização da focalização como ferramenta de alívio à pobreza é admitir, em um primeiro momento, a hipótese de que seja possível discriminar exatamente quem é pobre e quem não é pobre.

Considerando que haja interesse em maximizar a redução da pobreza na presença de um orçamento limitado, deve-se decidir qual o número de beneficiários que será coberto pelo programa e qual o nível de transferência (Coady et al., 2004). Nesse sentido, a discussão sobre como identificar a população-alvo é relevante.

\section{O TESTE DE ELEGIBILIDADE MULTIDIMENSIONAL \\ (PROXY MEANS TEST)}

Existem vários métodos para definir o público-alvo de políticas públicas de transferência de renda, dentre os quais se destacam: Método Categórico; Auto-seleção; Teste de Renda Verificada; Teste de Renda Não Verificada e Teste de Elegibilidade Multidimensional. ${ }^{3}$ O Teste de Elegibilidade Multidimensional permite indentificar a população em condição de pobreza com 
base em características individuais ou domiciliares correlacionadas com a renda. Os dados a serem trabalhados pelo método podem ser obtidos através de censos ou pesquisas domiciliares que investigam características variadas sobre os indivíduos e seus domicílios. ${ }^{4} \mathrm{O}$ objetivo do método é, a partir das informações estatísticas sobre indivíduos e domicílios, aplicar modelagem estatística ${ }^{5}$ e construir indicadores que serão utilizados para ordenar as famílias ou domicílios, permitindo a classificação de pobres ou não-pobres.

Antes de esse método ser implementado, deve previamente ter sido decidido qual a forma que será utilizada para inscrição no programa social — se entrevistas; por demanda; ou uma combinação desses procedimentos. O resultado deve gerar um cadastro para a seleção de beneficiários que possa ser atualizado, considerando que algumas variáveis podem perder o poder de prever a pobreza ou de discriminar pobres de não-pobres. ${ }^{6}$

Os custos administrativos da aplicação do Teste de Elegibilidade Multidimensional, em princípio, são menores do que os de outros métodos, como, por exemplo, os necessários para implementar o Teste de Renda Verificada. Como não exige comprovação de renda, esse procedimento é menos suscetível aos incentivos adversos ao trabalho.

Uma das principais vantagens de sua utilização é tornar o procedimento replicável, isto é, a aplicação do Teste de Elegibilidade Multidimensional torna possível que domicílios semelhantes (pelo menos no que se refere às variáveis escolhidas) recebam o mesmo tratamento ou decisão, mesmo se avaliados por membros diferentes do grupo em dias diferentes. Constitui, portanto, ferramenta importante para evitar a corrupção ou politização do programa.

As desvantagens do procedimento são: não considerar algumas circunstâncias especiais do domicílio; a função obtida através de modelagem estatística é, em geral, desenhada para funcionar bem em média, mas não para categorizar cada domicílio individualmente; o procedimento exige o treinamento de pessoal para a utilização das fórmulas; e, por último, algumas pessoas podem considerar o procedimento arbitrário por não compreenderem os métodos estatísticos empregados.

Esse método foi adotado em vários países da América Latina como, por exemplo, Chile (Ficha Cas), Costa Rica, Colômbia (Sisben) e México (Progresa) (Legovini, 1999). Outros países da América Latina que estão desen- 
volvendo sistemas de focalização utilizando o Teste de Elegibilidade Multidimensional são a Argentina, o Equador, a Jamaica, Honduras e a Nicarágua. Armênia, Indonésia e Turquia são exemplos de países fora da América Latina que também utilizam esse método (Coady et al., 2004).

Uma aplicação desse método no Brasil, utilizando os dados da PNAD 1998, é encontrada no trabalho de Anuatti Neto, Fernandes e Pazello (2000).

\section{UMA MEDIDA DE DESEMPENHO DA FOCALIZAÇÃO}

Na prática, os gerenciadores dos programas sociais não possuem informação perfeita sobre quem se encontra em condição de pobreza. Portanto, considerando que a elegibilidade do programa é baseada em informação imperfeita, é inevitável a presença de algum erro de inclusão (identificar como pobres pessoas que são não-pobres e admiti-las no programa), e/ou erros de exclusão (identificar como não-pobres pessoas que são pobres e não admiti-las no programa). Caso os recursos do programa fossem ilimitados, tais erros poderiam ser minimizados através da coleta de informação adicional. Entretanto, em um mundo de recursos limitados, os formuladores de políticas e os gerenciadores dos programas precisam saber se tais custos são justificados pela real melhoria na focalização. Além disso, os governantes e a sociedade precisam conhecer o quão efetiva é uma dada intervenção focalizada. Isso requer uma medida de desempenho da focalização.

Legovini (1999) descreve três critérios para a avaliação do método de focalização: a eficiência da focalização, o vazamento do programa e os custos administrativos envolvidos. Para compreender esses critérios, é importante notar que, qualquer que seja o método escolhido, ele está sujeito a dois tipos de erro, análogos aos erros do tipo I e do tipo II da inferência estatística. Esses erros são conhecidos na literatura como erros de focalização. São eles: excluir do programa famílias que deveriam ser incluídas (erro tipo I, erro de cobertura ou erro de exclusão) e incluir no programa famílias que não deveriam ser incluídas (erro tipo II, vazamento ou erro de inclusão), conforme o esquema da tabela 1 . Um método de focalização é dito eficiente quando minimiza o erro do tipo I (Cornia e Stewart, 1995).

O ponto de equilíbrio desejado entre o custo de vazamento e os custos administrativos deve ser avaliado, assim como a possibilidade de que pro- 
Tabela 1: Decisão sobre a inclusão ou exclusão de indivíduos em um programa social

\begin{tabular}{lll}
\hline Ação & Pobre & Situação \\
& Não-pobre \\
\hline Incluir no Programa & Decisão correta & Erro tipo II \\
Excluir do Programa & Erro tipo I & Decisão correta \\
\hline
\end{tabular}

gramas com um certo nível de vazamento possuam uma capacidade maior de se manterem que programas sem nenhum vazamento, devido a uma maior necessidade de suporte operacional.

De uma forma geral, é desejado que a focalização seja realizada de maneira a minimizar os dois tipos de erros citados. À medida que o programa se expande, a tendência é diminuir o erro do tipo I e aumentar o erro do tipo II. O inverso ocorreria com uma redução do programa. A maneira de reduzir os dois tipos de erros é melhorar a capacidade de discriminação, o que, por sua vez, aumenta os custos administrativos do programa (Anuatti Neto et al., 2000).

Considerando que se deseja minimizar os dois tipos possíveis de erros de focalização: Anuatti Neto, Fernandes e Pazello (2000) sugerem o seguinte indicador de focalização IF: IF $=\alpha\left[P_{I}-P_{E}\right]+(1-\alpha)\left[N P_{E}-N P_{I}\right]$, que varia no intervalo $[-1,1]$, e quanto mais próximo de um, melhor a focalização; $\alpha$ é o fator de ponderação $(0 \leq \alpha \leq 1)$ que permite escolher entre priorizar a eficiência do programa ou o controle do vazamento deste; $P_{I}$ é a proporção de domicílios pobres devidamente incluídos no programa; $P_{E}$ é a proporção de domicílios pobres indevidamente excluídos do programa; $N P_{E}$ é a proporção de domicílios não-pobres devidamente excluídos do programa; $N P_{I}$ é a proporção de domicílios não-pobres indevidamente incluídos no programa; $\left[P_{I}-P_{E}\right]$ representa a eficiência no alcance da política; e $\left[N P_{E}-N P_{I}\right]$ representa uma medida do vazamento do programa.

Ao ajustar um modelo para estimar a probabilidade de um domicílio estar em condição de pobreza segundo suas características, o indicador é calculado considerando que são incluídos no programa os domicílios para os quais as probabilidades previstas $\hat{p}_{i}$ de ocorrência do evento (condição de pobreza) para o $i$-ésimo domicílio são $\hat{p}_{i} \geq \frac{(1-\alpha) P O B}{\alpha N P O B+(1-\alpha) P O B}$, sendo $P O B$ o número de pobres segundo a linha de pobreza e $N P O B$ o número de não-pobres segundo a linha de pobreza. Em seguida, escolhe-se o valor de $\alpha$ 
e calcula-se a proporção de domicílios pobres incluídos e a proporção de domicílios não-pobres incluídos. Quando $\alpha=1$, então $I F=\left[P_{I}-P_{E}\right]$, ou seja, apenas o critério de inclusão de pobres é considerado. Nesse caso, IF seria máximo, por exemplo, através da universalização dos benefícios.

Uma deficiência desse indicador é não considerar a intensidade da pobreza, ou seja, a exclusão do programa de um domicílio pobre, com renda próxima à linha de pobreza, produz o mesmo impacto no indicador proposto do que a exclusão de outra família mais pobre. Por outro lado, a inclusão de um domicílio não pobre com renda próxima à linha de pobreza produz o mesmo impacto no indicador do que a inclusão de um domicílio rico. Entretanto, é possível considerar a intensidade da pobreza realizando uma ponderação baseada na distância entre a renda domiciliar per capita e a linha de pobreza. Quanto maior a distância, maior o peso.

Os autores definem o peso $\phi_{i}=\frac{y_{i}}{E\left(y_{i} \mid Y_{i}=0 ; 1\right)}$, no qual $R_{i}$ é a renda domiciliar per capita do $i$-ésimo domicílio; $y_{i}=\left|L-R_{i}\right|$ é a distância da renda do domicílio à linha de pobreza $L ; E\left(y_{i} \mid Y_{i}=0 ; 1\right)$ é a distância média dos domicílios pobres e não-pobres à linha de pobreza; e $Y_{i}$ é uma variável que assume o valor 1 quando o domicílio encontra-se em condição de pobreza, sendo igual a zero, caso contrário.

\section{APLICAÇÃO DO TESTE DE ELEGIBILIDADE MULTIDIMENSIONAL}

Como apresentado na seção 3, a aplicação do Teste de Elegibilidade Multidimensional requer a análise de características individuais ou domiciliares correlacionadas com a renda, utilizando métodos estatísticos. Neste artigo são apresentados os resultados da aplicação do método utilizando dados coletados na PNAD 2003.

Como a PNAD só dispõe da renda do indivíduo no mês da pesquisa, as pessoas desocupadas no período de referência da pesquisa declaram renda nula, o que poderia acarretar famílias de alto padrão de vida classificadas como pobres, caso sua renda nula fosse diretamente comparada à linha de pobreza. Ou seja, é possível que a renda de alguns indivíduos seja nula apenas no mês de referência da pesquisa (ou em um período curto que inclui o 
mês de referência da pesquisa), por uma questão conjuntural, e, ainda assim, a família não seja, de fato, estruturalmente pobre.

Nota-se que, na PNAD 2003, existem 179.845 pessoas de 10 anos ou mais com renda nula (47,9\% da amostra). O número de domicílios na amostra da PNAD 2003 com renda nula é 1.657 (1,6\% da amostra). Portanto, antes de realizar o ajustamento do modelo de regressão logística, optou-se por imputar um valor de renda para todos os desocupados.

O procedimento consistiu em separar os indivíduos em três categorias: ocupados, desocupados e fora da força. Foi imputado um valor de renda para todos os indivíduos desocupados cuja renda total informada na PNAD 2003 era igual à zero. A equação utilizada para estimar a renda desses indivíduos foi obtida em um estudo recente realizado por Pinto de Carvalho (2005). Vale ressaltar que nesse estudo o referido autor utilizou os procedimentos adequados de modelagem estatística para pesquisas amostrais complexas, isto é, a estimação do modelo foi realizada considerando as diversas características do plano amostral utilizado na coleta dos dados, conforme procedimento descrito em Pessoa e Silva (1998).

Após o procedimento de imputação de renda, foi ajustado um modelo de regressão logística para identificar como determinadas características familiares/domiciliares estão relacionadas à ocorrência da pobreza, isto é, como auxiliam na discriminação da população em dois grupos: pobres e não-pobres. $\mathrm{O}$ evento de interesse é descrito por uma variável aleatória indicadora que assume o valor um se o domicílio encontra-se em condição de pobreza e é igual a zero, caso contrário.

Para a seleção das variáveis explicativas, foram consideradas todas aquelas apontadas em Anuatti Neto, Fernandes e Pazello (2000) e, além destas, outras utilizadas na literatura para medir o risco de pobreza (maiores detalhes em Faria, 2006). De acordo com Pessoa e Silva (1998), considerando que o plano amostral utilizado na PNAD é complexo (usa estratificação, conglomeração e probabilidades desiguais de seleção), o método adequado para a estimação dos parâmetros do modelo é o Método da Máxima Pseudoverossimilhança. Portanto, o procedimento de estimação do modelo incorpora as características do plano amostral da PNAD no cálculo das estimativas dos parâmetros e respectivas variâncias. As probabilidades estimadas pelo modelo $\left(\hat{p}_{i}\right)$ fornecem o valor do escore de propensão (probabilidade 
de o domicílio estar em condição de pobreza de acordo com suas características socioeconômicas).

Inicialmente, foram ajustados quatro modelos. No primeiro modelo, o único fator relacionado a características do domicílio foi o acesso à telefonia, pois, ao incluir essa variável, todos os demais fatores relacionados a características domiciliares perderam o poder de discriminar pobres de não-pobres (deixaram de ser significantes no modelo). Entretanto, de acordo com relatório elaborado pela Divisão Estatística das Nações Unidas, condições relacionadas a abrigo, esgotamento e abastecimento de água em condições adequadas são consideradas fundamentais ao bem-estar das famílias. Portanto, decidiu-se ajustar um segundo modelo sem o fator acesso à telefonia. Procedendo dessa forma, três características domiciliares (material resistente, acesso à água e acesso a esgotamento em condições adequadas) foram significantes no modelo. O terceiro modelo ajustado corresponde ao primeiro modelo acrescido de todas as interações significantes, e o quarto modelo, ao segundo modelo ajustado mais interações significantes, tendo sido todas as interações possíveis avaliadas. Entretanto, não foram observadas explicações importantes que justificassem a inclusão das interações nos modelos.

Para todos esses quatro modelos, a variável resposta foi definida considerando como pobres as pessoas que residiam em domicílios com renda domiciliar per capita abaixo das linhas de pobreza regionalizadas propostas por Rocha (2003). Adicionalmente, ajustou-se um quinto modelo, para o qual foram consideradas pobres as pessoas que residiam em domicílios com renda domiciliar per capita abaixo de meio salário mínimo.

Observou-se que, neste último modelo, todas as características relacionadas à estrutura do domicílio foram significantes, inclusive a posse de telefone no domicílio. Além disso, para esse, os indicadores de focalização foram melhores em todas as regiões. Portanto, esse foi o modelo escolhido para ser analisado de forma mais detalhada. ${ }^{7}$

Como neste modelo, a variável resposta utilizada foi construída de forma não regionalizada; constata-se que são significantes e relevantes os fatores região (Norte, Nordeste, Sul, Sudeste e Centro-Oeste) e área (urbana e rural), além de todas as características relacionadas à estrutura do domicílio consideradas. A tabela 2 apresenta as estimativas dos coeficientes do modelo escolhido e a significância dos testes de hipótese de nulidade de cada efeito. 
Tabela 2: Coeficientes estimados para o modelo escolhido

\begin{tabular}{|c|c|c|c|c|c|}
\hline Efeitos & iciente & $p$-valor & Efeitos & Coeficiente & $p$-valor \\
\hline Intercepto & $-5,59$ & 0 & & & \\
\hline \multicolumn{3}{|l|}{ Região } & \multicolumn{3}{|l|}{ Idade da pessoa de referência } \\
\hline Sul & \multicolumn{2}{|l|}{0} & Menos que 25 anos & 1,41 & 0 \\
\hline Sudeste & 0,09 & 0,0573 & 25 a 34 anos & 0,98 & 0 \\
\hline Centro-Oeste & 0,2 & 0,0007 & 35 a 44 anos & 0,81 & 0 \\
\hline Norte & 0,27 & 0 & 45 a 54 anos & 0,65 & 0 \\
\hline Nordeste & 0,99 & 0 & 55 anos ou mais & 0 & \\
\hline \multirow{2}{*}{\multicolumn{3}{|c|}{ Área }} & \multicolumn{3}{|l|}{ Esgoto } \\
\hline IIrhan & & & Sim & 0 & \\
\hline Urbana & \multicolumn{2}{|l|}{0} & Não & 0,36 & 0 \\
\hline Rural & \multirow[t]{2}{*}{0,19} & \multirow[t]{2}{*}{0,0002} & \multicolumn{3}{|l|}{ Lixo } \\
\hline Tipo de família & & & Sim & 0 & \\
\hline Chefe e cônjuge recentes & 0 & & Não & 0,25 & 0 \\
\hline Chefe homem sem cônjuge & $-0,33$ & 0 & Telefone & & \\
\hline Chefe mulher sem cônjuge & 0,31 & 0 & Sim & 0 & \\
\hline \multicolumn{3}{|l|}{ Número de crianças } & Não & 1,18 & 0 \\
\hline 0 & \multicolumn{2}{|l|}{0} & \multicolumn{3}{|l|}{ Luz } \\
\hline 1 & 0,82 & 0 & Sim & 0 & \\
\hline 2 & 1,47 & 0 & Não & 0,18 & 0,0015 \\
\hline 3 & 2,1 & 0 & Material resistente & & \\
\hline 4 ou mais & 2,67 & 0 & Sim & 0 & \\
\hline \multirow{2}{*}{\multicolumn{3}{|c|}{ Anos de estudo }} & Não & 0,21 & 0,0001 \\
\hline & & & \multicolumn{3}{|l|}{ Água } \\
\hline 0 a 3 anos & 1,43 & 0 & $\operatorname{sim}$ & 0 & \\
\hline 4 anos & 1,11 & 0 & Não & 0,29 & 0 \\
\hline 5 a 7 anos & 0,81 & 0 & Densidade morador-cômodo & 0,58 & 0 \\
\hline 8 anos ou mais & 0 & & Razão de pendência & 0,21 & 0 \\
\hline
\end{tabular}

\subsection{Análise do modelo escolhido}

O modelo para descrever a probabilidade de um domicílio estar em condição de pobreza é dado por:

$$
\begin{aligned}
& \ln \left(\frac{P_{\text {tujklmnopqrsi }}}{1-P_{\text {tujklmnopqrsi }}}\right)=\beta_{0+} \beta_{t+}^{\text {reg }} \beta_{u+}^{\text {area }} \beta_{j+}^{\text {fam }} \beta_{k+}^{\text {cri }} \beta_{l+}^{\text {esc }} \beta_{m+}^{\text {ida }} \beta_{n+}^{\text {esg }} \beta_{o+}^{\text {lixo }} \beta_{p+}^{\text {tel }} \beta_{q+}^{\text {luz }} \\
& \beta_{r}^{\text {mat }}+\beta_{s}^{\text {agua }} \hat{a}^{\text {dens }} x_{1 i}+\hat{a}^{\text {dep }} x_{2 i} \text {, sendo: }
\end{aligned}
$$

$\beta_{t}^{\text {reg }}$ o efeito da t-ésima região (Sul, Sudeste, Centro-Oeste, Norte e Nordeste; $t=1,2,3,4,5$ 
$\beta_{u}^{\text {area }}$ o efeito da $u$-ésima área (urbana e rural); $u=1,2$

$\beta_{j}^{\text {fam }}$ o efeito do $j$-ésimo tipo de família; $j=1,2,3$ $\beta_{k}^{c r i} \quad \begin{aligned} & \text { o efeito do número de crianças menores que } 14 \text { anos presentes no domicílio; } \\ & k=1,2,3,4,5\end{aligned}$

$\beta_{l}^{e s c}$ o efeito dos anos de escolaridade da pessoa de referência no domicílio; $l=1,2,3,4$

$\beta_{m}^{i d a}$ o efeito da idade da pessoa de referência no domicílio; $m=1,2,3,4,5$

$\beta_{n}^{\text {esg }}$ o efeito do acesso a esgotamento adequado; $n=1,2$

$\beta_{o}^{\text {lixo }}$ o efeito da coleta de lixo; $o=1,2$

$\beta_{p}^{\text {tel }}$ o efeito do acesso à telefonia; $p=1,2$

$\beta_{q}^{\text {luz }}$ o efeito do acesso à luz; $q=1,2$

$\beta_{r}^{\text {mat }}$ o efeito de a construção do domicílio ser de material resistente; $r=1,2$

$\beta_{s}^{\text {água }}$ o efeito do acesso à água em condições adequadas; $s=1,2$

$x_{1 i} \quad$ a densidade morador/cômodo do $i$-ésimo domicílio

$x_{2 i} \quad$ a razão de dependência do $i$-ésimo domicílio

Os resultados da tabela 2 indicam que os coeficientes crescem de acordo com o número de crianças com menos de 14 anos no domicílio e quanto menores forem os anos de estudo e a idade do chefe do domicílio. $O$ fato de não possuir esgotamento adequado, coleta de lixo adequada, telefone, luz, material resistente e água em condições adequadas aumenta a probabilidade de o domicílio ser classificado como pobre. Em relação ao tipo de família, o coeficiente mais alto ocorre quando a pessoa de referência no domićlio é mulher sem cônjuge. $\mathrm{O}$ coeficiente é superior na área rural. Quanto às regiões, o coeficiente mais elevado é o referente à região Nordeste, seguido das regiões Norte, Centro-Oeste, Sudeste e Sul. 
Tabela 3: Estatística de Wald para avaliar a quantidade do ajuste do modelo

\begin{tabular}{|c|c|c|c|c|c|c|c|}
\hline & G.L. & Wald F & p-valor & & G.L. & Wald F & p-valor \\
\hline Modelo completo & 27 & 788,99 & 0 & & & & \\
\hline Modelo menos intercepto & 26 & 579,83 & 0 & Esgotamento adequado & 1 & 142,49 & 0 \\
\hline Intercepto & & & & Coleta de lixo adequada & 1 & 26,04 & 0 \\
\hline Região & 4 & 200,77 & 0 & Acesso à telefonia & 1 & 2053,54 & 0 \\
\hline Área & 1 & 14,33 & 0,0002 & Acesso à energia elétrica & 1 & 10,12 & 0,0015 \\
\hline Tipo de família & 2 & 93,29 & 0 & $\begin{array}{l}\text { Material de construção } \\
\text { resistente }\end{array}$ & 1 & 15,26 & 0,0001 \\
\hline $\begin{array}{l}\text { Número de crianças entre } \\
0 \text { e } 14 \text { anos }\end{array}$ & 4 & 397,99 & 0 & Água adequada & 1 & 46,86 & 0 \\
\hline $\begin{array}{l}\text { Anos de estudo da } \\
\text { pessoa de referência }\end{array}$ & 3 & 551,83 & 0 & Densidade morador-cômodo & 1 & 324,16 & 0 \\
\hline $\begin{array}{l}\text { Idade da pessoa } \\
\text { de referência }\end{array}$ & 4 & 218,71 & 0 & Razão de dependência & 1 & 75,05 & 0 \\
\hline
\end{tabular}

Para avaliar a qualidade do ajuste do modelo, foi elaborada a tabela 3 , que apresenta a estatística de Wald para cada variável/fator incluído no modelo.

Os resultados demonstram que todas as variáveis/fatores incluídos nesse modelo são significantes, considerando um nível de significância igual a 0,05 .

\subsubsection{Gráficos das probabilidades estimadas}

A partir do ajuste do modelo, foi possível estimar as probabilidades de ocorrência do evento (condição de pobreza) para categorias de interesse. Portanto, foram elaborados gráficos que descrevem a probabilidade estimada de um domicílio estar em condição de pobreza condicionada a variações na razão de dependência (gráfico 1), no número de crianças do domicílio (gráfico 2) e nos anos de estudo da pessoa de referência do domicílio (gráfico 3), somente para a região Nordeste, que corresponde ao nível do fator região, cujo coeficiente estimado pelo modelo é o mais elevado. Os gráficos para as demais regiões do País estão disponíveis em Faria (2006).

O gráfico 1 apresenta a probabilidade estimada condicionada a variações na razão de dependência para 10 situações diferentes, cujo resumo encontra-se no quadro 1 . A densidade morador-cômodo foi fixada em sua mediana (igual a 0,6), e considerou-se que o domicílio possui esgotamento, coleta de lixo, água em condições adequadas, acesso à luz e à telefonia e material de construção resistente. 
Quadro 1: Situações consideradas para análise das probabilidades estimadas segundo variações da razão de dependência e do número de crianças do domicílio

\begin{tabular}{|c|c|c|c|c|c|c|c|c|c|c|c|}
\hline \multirow[b]{3}{*}{ Área } & \multirow[b]{3}{*}{ Urbana } & \multicolumn{10}{|c|}{ Situação } \\
\hline & & 1 & 2 & 3 & 4 & 5 & 6 & 7 & 8 & 9 & 10 \\
\hline & & $\square$ & & $\square$ & & $\square$ & & $\square$ & & $\square$ & \\
\hline & Rural & & $\square$ & & $\square$ & & $\square$ & & $\square$ & & $\square$ \\
\hline \multicolumn{12}{|c|}{ Tipo de família } \\
\hline & Chefe e cônjuge presentes & $\square$ & $\square$ & $\square$ & $\square$ & $\square$ & $\square$ & & & & \\
\hline & Chefe homem sem cônjuge & & & & & & & & & & \\
\hline & Chefe mulher sem cônjuge & & & & & & & $\square$ & $\square$ & $\square$ & $\square$ \\
\hline \multicolumn{12}{|c|}{ Número de crianças } \\
\hline & 0 & $\square$ & $\square$ & & & & & & & & \\
\hline & 1 & & & $\square$ & $\square$ & & & & & & \\
\hline & 2 & & & & & $\square$ & $\square$ & & & & \\
\hline & 3 & & & & & & & $\square$ & $\square$ & & \\
\hline & 4 ou mais & & & & & & & & & $\square$ & $\square$ \\
\hline \multicolumn{12}{|c|}{ Anos de estudo } \\
\hline & 0 a 3 anos & & & & & & & & & $\square$ & $\square$ \\
\hline & 4 anos & & & & & $\square$ & $\square$ & $\square$ & $\mathbf{\square}$ & & \\
\hline & 5 a 7 anos & & & $\square$ & $\square$ & & & & & & \\
\hline & 8 anos ou mais & $\square$ & $\square$ & & & & & & & & \\
\hline \multicolumn{12}{|c|}{ Idade } \\
\hline & Menos que 25 anos & & & & & & & & & 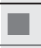 & $\square$ \\
\hline & 25 a 34 anos & & & & & & & $\square$ & $\square$ & & \\
\hline & 35 a 44 anos & & & & & $\square$ & $\square$ & & & & \\
\hline & 45 a 54 anos & & & $\square$ & $\square$ & & & & & & \\
\hline & 55 anos ou mais & 口 & $\mathbf{0}$ & & & & & & & & \\
\hline
\end{tabular}

O gráfico mostra que a probabilidade de estar em condição de pobreza aumenta conforme aumenta a razão de dependência para todas as situações consideradas. Para as situações 1 e 2 , as probabilidades estimadas de ocorrência do evento são sempre inferiores a 0,1 , independentemente do valor da razão de dependência. Por outro lado, considerando as situações 7, 8, 9 e 10, as probabilidades estimadas de ocorrência do evento de interesse são sempre superiores a 0,5. Observa-se ainda que, para qualquer uma das situações consideradas, as probabilidades estimadas para áreas rurais são mais elevadas que as probabilidades estimadas para a área urbana.

O gráfico 2 apresenta a probabilidade estimada de um domicílio estar em condição de pobreza condicionada a variações no número de crianças no domicílio. As situações consideradas são idênticas às utilizadas no gráfi- 


\section{Gráfico 1: Probabilidades estimadas por razão de dependência - região Nordeste}
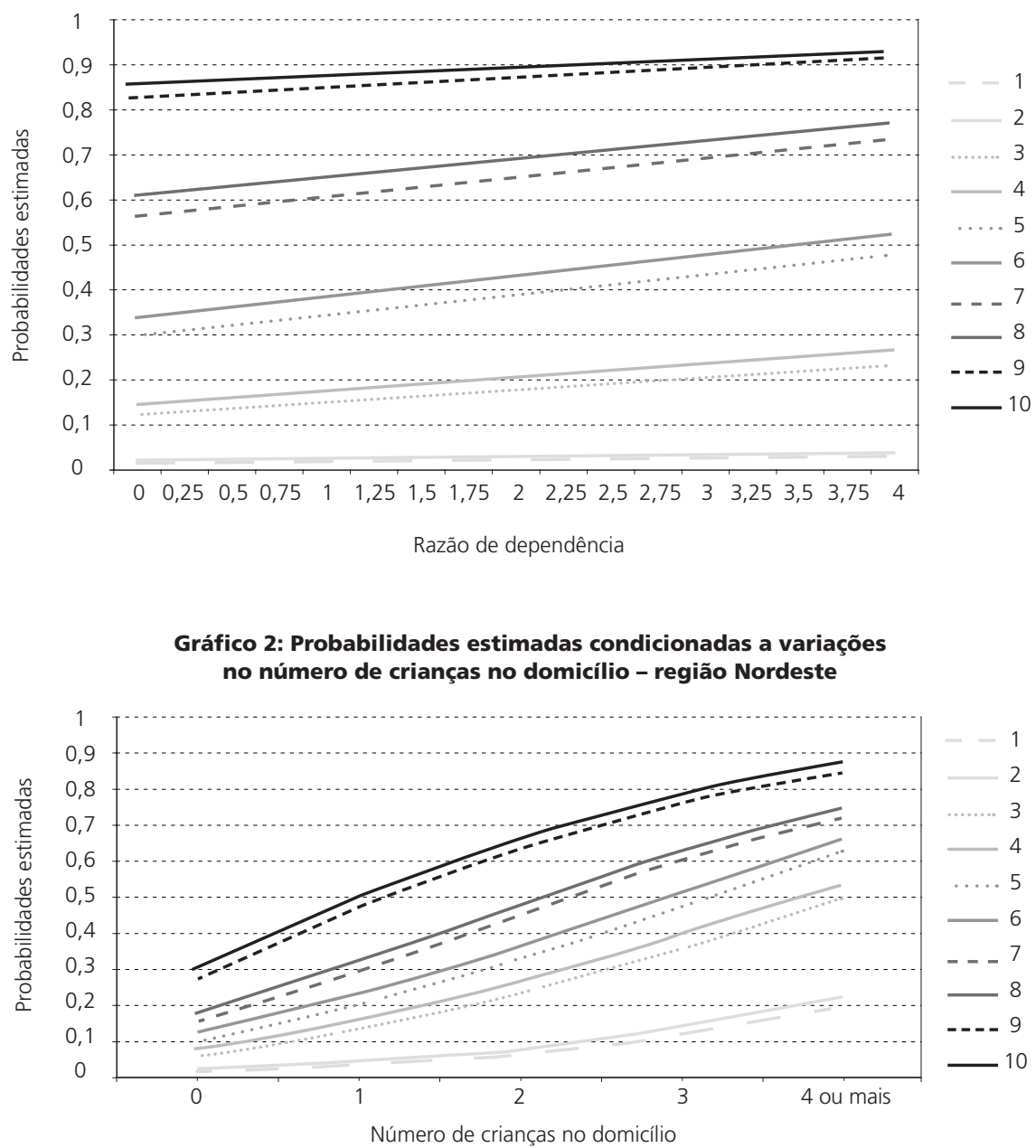

co anterior (representadas no quadro 1), porém a razão de dependência foi fixada em sua mediana $(0,4)$, e a variação de interesse ocorre no fator número de crianças no domicílio.

Observa-se que a probabilidade de um domicílio estar em condição de pobreza aumenta conforme aumenta o número de crianças no domicílio, para todas as situações consideradas, e a probabilidade é sempre superior na área rural em relação à área urbana, para qualquer situação e qualquer que seja o número de crianças no domicílio. 


\section{Gráfico 3: Probabilidades estimadas condicionadas a variações nos anos de estudo da pessoa de referência do domicílio - região Nordeste e área rural}

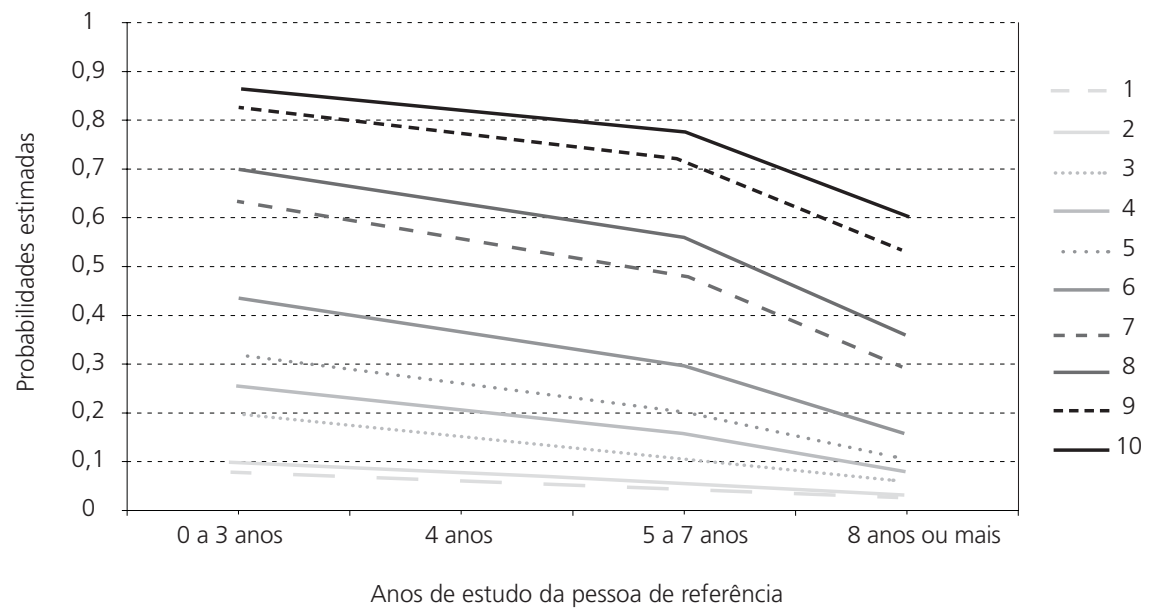

O gráfico mostra que a probabilidade estimada de um domicílio estar em condição de pobreza quando não há crianças no domicílio é, no máximo, igual a 0,3 (situação 10). Por outro lado, quando há quatro ou mais crianças no domicílio, a probabilidade de estar em condição de pobreza para essa mesma situação é superior a 0,85. Exceto para as situações 1 e 2 , a probabilidade estimada de ocorrência do evento é igual ou superior a 0,5 sempre que o domicílio possui quatro ou mais crianças.

Para avaliar as variações das probabilidades estimadas de um domicílio estar em condição de pobreza, condicionada às variações de anos de estudo, são consideradas as 10 situações resumidas no quadro 2 , tendo sido a razão de dependência e a densidade morador-cômodo fixadas em suas medianas (0,4 e 0,6 , respectivamente).

O gráfico 3, que descreve a probabilidade estimada de um domicílio estar em condição de pobreza condicionada a variações nos anos de estudo da pessoa de referência no domicílio, foi elaborado considerando a região Nordeste e a área rural, que são os níveis dos fatores região e área cujos coeficientes estimados pelo modelo são mais elevados. Para essa análise, considerou-se também que o domicílio possui esgotamento, coleta de lixo, água em condições adequadas, acesso à luz e à telefonia e material de construção resistente. A probabilidade de ser pobre diminui com os anos de estudo e, 
Quadro 2: Descrição das situações consideradas na elaboração do gráfico 3

\begin{tabular}{|c|c|c|c|c|c|c|c|c|c|c|c|}
\hline & & \multicolumn{10}{|c|}{ Situação } \\
\hline & & 1 & 2 & 3 & 4 & 5 & 6 & 7 & 8 & 9 & 10 \\
\hline \multicolumn{12}{|l|}{ Tipo de família } \\
\hline & Chefe e cônjuge presentes & $\square$ & & $\square$ & & $\square$ & & $\square$ & & $\square$ & \\
\hline & Chefe homem sem cônjuge & & & & & & & & & & \\
\hline & Chefe mulher sem cônjuge & & $\square$ & & $\square$ & & $\square$ & & $\square$ & & $\square$ \\
\hline \multicolumn{12}{|l|}{ Número de crianças } \\
\hline & 0 & $\square$ & $\square$ & & & & & & & & \\
\hline & 1 & & & $\square$ & $\square$ & & & & & & \\
\hline & 2 & & & & & $\square$ & 口 & & & & \\
\hline & 3 & & & & & & & $\square$ & $\square$ & & \\
\hline & 4 ou mais & & & & & & & & & $\square$ & $\square$ \\
\hline \multicolumn{12}{|l|}{ Idade } \\
\hline & Menos que 25 anos & & & & & & & & & $\square$ & $\square$ \\
\hline & 25 a 34 anos & & & & & & & $\square$ & $\square$ & & \\
\hline & 35 a 44 anos & & & & & $\square$ & $\square$ & & & & \\
\hline & 45 a 54 anos & & & $\square$ & $\square$ & & & & & & \\
\hline & 55 anos ou mais & $\square$ & $\mathbf{\square}$ & & & & & & & & \\
\hline
\end{tabular}

além disso, pode-se notar que a probabilidade estimada é sempre superior quando a pessoa de referência no domicílio é mulher, sem cônjuge presente, uma vez fixadas as demais condições.

\subsubsection{Razões de vantagens}

Além da análise gráfica das probabilidades estimadas, foram estimadas as vantagens de ocorrência do evento de interesse em relação à não-ocorrência desse evento para o modelo escolhido. As tabelas a seguir apresentam as razões de vantagens para os fatores do modelo, lembrando que essas estatísticas são úteis para comparar níveis dos fatores. Quando a razão de vantagem é menor do que um, o nível utilizado como referência possui maior vantagem em favor de ser pobre.

O nível utilizado como referência foi a região Sul. A vantagem em favor da situação de pobreza é 170\% maior nos domicílios situados na região Nordeste do que nos domicílios da região Sul. A comparação entre as vantagens para os domicílios de áreas rurais e urbanas (qualquer das regiões) indica que a vantagem em favor de ser pobre é $20 \%$ superior em áreas rurais do País do que em áreas urbanas. 
Tabela 4: Razões de vantagens por região

\begin{tabular}{ll}
\hline Macrorregião & \\
\hline Sudeste/Sul & 1,09 \\
\hline Centro-Oeste/Sul & 1,22 \\
\hline Norte/Sul & 1,31 \\
\hline Nordeste/Sul & 2,7 \\
\hline
\end{tabular}

Tabela 5: Razões de vantagens para o número de crianças no domicílio

\begin{tabular}{lc}
\hline Número de crianças de 0 a 14 anos no domicílio \\
\hline uma/nenhuma & 2,28 \\
\hline duas/nenhuma & 4,34 \\
\hline três/nenhuma & 8,16 \\
\hline quatro ou mais/nenhuma & 14,48 \\
\hline
\end{tabular}

Tabela 6: Razões de vantagens para o fator anos de estudo da pessoa de referência do domicílio

Anos de estudo da pessoa de referência

\begin{tabular}{ll}
\hline 0 a 3 anos/8 anos ou mais & 4,16 \\
\hline 4 anos/8 anos ou mais & 3,03 \\
\hline 5 a 7 anos/8 anos ou mais & 2,25 \\
\hline
\end{tabular}

A vantagem em favor da ocorrência de situação de pobreza cresce conforme aumenta o número de crianças menores que 14 anos no domicílio (fixando como nível de referência o domicílio não possuir crianças menores que 14 anos de idade). Nos domicílios que possuem uma criança menor que 14 anos, a vantagem em favor da ocorrência da condição de pobreza em relação aos domicílios que não possuem crianças menores que 14 anos é $128 \%$ maior.

Nesse caso, as razões de vantagens em relação ao nível de base (pessoa de referência com oito anos ou mais de estudo) diminuem conforme aumentam os anos de estudo da pessoa de referência. O resultado está de acordo com o esperado: a probabilidade de ser pobre é mais alta para domicílios cuja pessoa de referência possui baixa escolaridade.

\section{RESULTADOS DA APLICAÇÃO DO INDICADOR DE FOCALIZAÇÃO}

Utilizando o fator de ponderação de $\alpha=0,5$, o indicador $I F=\left[P_{I}-N P_{I}\right]$ permite avaliar a diferença nas probabilidades de inclusão das famílias/do- 
micílios pobres e não-pobres no programa considerando-se somente a capacidade de discriminação. Utilizando o critério de inclusão apresentado na seção 5 , foram obtidos os resultados apresentados na tabela 7 .

Nesse caso, observa-se que 33\% dos domicílios são incluídos no programa no País todo. O percentual mais elevado de incluídos ocorreu na região Nordeste, com $45 \%$ de incluídos. Nota-se, também, que o percentual de domicílios incluídos no programa foi superior à proporção de pobres em todas as regiões do País, e o percentual de domicílios pobres incluídos variou entre 75\% (nas regiões Sudeste, Centro-Oeste e Norte) e 80\% (na região Sul).

Aumentar o fator de ponderação de $\alpha=0,5$ para $\alpha=0,7$ significa dar maior peso à eficiência do alcance do programa do que à medida de vazamento deste. Ao utilizar o fator $\alpha=0,7$, a tabela 8 mostra que a proporção de domicílios pobres incluídos e a proporção de domicílios não-pobres incluídos no programa foram superiores em todas as regiões. $\mathrm{O}$ indicador de focalização aumenta em relação aos calculados com $\alpha=0,5$, em todas as regiões, antes e após a aplicação do critério de reponderação.

\section{CONCLUSÕES}

Este artigo destacou que, quando a focalização de políticas sociais é considerada a opção mais indicada no combate à pobreza e desigualdade social, a análise dos custos e dos benefícios envolvidos na aplicação dessas políticas deve ser realizada criteriosamente, considerando as características socioeconômicas e culturais da população.

Nesse artigo, fez-se uma aplicação do Teste de Elegibilidade Multidimensional aos dados da PNAD 2003, com o objetivo de identificar o percentual de domicílios pobres por macrorregião do País. Nosso exercício apontou para um percentual de $22 \%$ de domicílios pobres no Brasil. Segundo a literatura internacional, o Teste de Elegibilidade Multidimensional apresenta bons resultados no sentido de eficiência. Esse método requer a aplicação de modelos estatísticos a dados coletados em pesquisas domiciliares por amostragem que combinam informações sobre renda com as demais características das famílias e dos domicílios na determinação de beneficiários de políticas públicas focalizadas. 
Tabela 7: Aplicação do indicador de focalização com $\alpha=\mathbf{0 , 5}$

\begin{tabular}{|c|c|c|c|c|c|c|c|c|}
\hline Macrorregiões & $\begin{array}{l}\text { Proporção } \\
\text { de domicílios } \\
\text { pobres }\end{array}$ & $\begin{array}{l}\text { Proporção } \\
\text { de domicílios } \\
\text { s incluídos } \\
\text { no programa }\end{array}$ & $P_{1}$ & $\mathrm{NP}_{\mathrm{I}}$ & $P_{E}$ & $\mathrm{NP}_{\mathrm{E}}$ & $\begin{array}{l}\text { Indicador de } \\
\text { focalização (IF) }\end{array}$ & $\begin{array}{l}\text { Indicador de } \\
\text { focalização } \\
\text { ponderado }\end{array}$ \\
\hline Sul & 0,13 & 0,31 & 0,80 & 0,24 & 0,20 & 0,76 & 0,57 & 0,77 \\
\hline Sudeste & 0,13 & 0,27 & 0,78 & 0,20 & 0,22 & 0,80 & 0,58 & 0,76 \\
\hline Centro-Oeste & 0,19 & 0,32 & 0,75 & 0,21 & 0,25 & 0,79 & 0,54 & 0,75 \\
\hline Norte & 0,30 & 0,37 & 0,75 & 0,21 & 0,25 & 0,79 & 0,54 & 0,74 \\
\hline Nordeste & 0,43 & 0,45 & 0,78 & 0,19 & 0,22 & 0,81 & 0,58 & 0,80 \\
\hline Brasil & 0,22 & 0,33 & 0,78 & 0,21 & 0,22 & 0,79 & 0,57 & 0,77 \\
\hline
\end{tabular}

Tabela 8: Aplicação do indicador de focalização com $\alpha=\mathbf{0 , 7}$

\begin{tabular}{lcccccccc}
\hline & \multicolumn{9}{c}{$\begin{array}{l}\text { Proporção } \\
\text { de domicílios } \\
\text { Macrorregiões }\end{array}$} & $\begin{array}{l}\text { de domicilios } \\
\text { incluídos } \\
\text { no programa }\end{array}$ & $\mathrm{P}_{\mathrm{I}}$ & $\mathrm{NP}_{\mathrm{I}}$ & $\mathrm{P}_{\mathrm{E}}$ & $\mathrm{NP}_{\mathrm{E}}$ & $\begin{array}{l}\text { Indicador de } \\
\text { focalização (IF) }\end{array}$ & $\begin{array}{l}\text { Indicador de } \\
\text { focalização } \\
\text { ponderado }\end{array}$ \\
\hline Sul & 0,13 & 0,47 & 0,90 & 0,41 & 0,10 & 0,59 & 0,62 & 0,79 \\
\hline Sudeste & 0,13 & 0,47 & 0,90 & 0,40 & 0,10 & 0,60 & 0,62 & 0,78 \\
\hline Centro-Oeste & 0,19 & 0,51 & 0,88 & 0,42 & 0,12 & 0,58 & 0,59 & 0,78 \\
\hline Norte & 0,30 & 0,54 & 0,88 & 0,39 & 0,12 & 0,61 & 0,59 & 0,78 \\
\hline Nordeste & 0,43 & 0,62 & 0,90 & 0,40 & 0,10 & 0,60 & 0,62 & 0,84 \\
\hline Brasil & 0,22 & 0,52 & 0,90 & 0,40 & 0,10 & 0,60 & 0,62 & 0,80 \\
\hline
\end{tabular}

A escolha das variáveis explicativas do modelo depende de um conjunto de fatores que a teoria econômica identifica como relevantes para descrever a probabilidade de um domicílio ser pobre, e da comprovação empírica da significância estatística desses fatores. O modelo de regressão logística aplicado neste artigo considerou como em condição de pobreza os domicílios cuja renda domiciliar per capita era inferior a meio salário mínimo.

No Teste de Elegibilidade Multidimensional, as probabilidades previstas pelo modelo de regressão constituem os escores de propensão à pobreza que, por sua vez, podem ser utilizados como critério de inclusão de domicílios em programas de transferência de renda. Assim, após a obtenção das estimativas dos escores de propensão, calculou-se uma medida de desempenho da focalização. Adicionalmente, observou-se que a utilização de um fator de ponderação, que atribui pesos diferenciados aos domicílios em função da distância entre a renda domiciliar per capita e a linha de pobreza, dando maior peso à eficiência do alcance do programa do que à sua medida de vazamento, implicou um aumento da medida de focalização em todas as cinco regiões do País. 


\section{NOTAS}

1. Ministério da Fazenda (2003) e Prefeitura da Cidade de São Paulo (2003).

2. Em geral, autores que defendem a focalização dos gastos argumentam que o problema dos programas sociais no Brasil não é de carência de recursos, mas da falta de qualidade na aplicação dos recursos (ver, por exemplo, Neri, 2003).

3. Para uma descrição mais detalhada, ver Faria (2006).

4. No Brasil, poderia ser sugerido como pesquisas particularmente úteis a Pesquisa Nacional por Amostra de Domicílios (PNAD) ou a Pesquisa de Orçamentos Familiares (POF).

5. É recomendado que sejam utilizadas poucas variáveis, de forma a assegurar sua validade e facilitar a interpretação dos métodos estatísticos empregados na população-alvo.

6. No caso do Brasil, poder-se-ia aplicar o método ao CadÚnico.

7. Os coeficientes estimados para todos os modelos ajustados podem ser encontrados em Faria (2006).

\section{REFERÊNCIAS BIBLIOGRÁFICAS}

ANUATTI NETO, F., FERNANDES, R., PAZELLO, E. T. (2000) "Políticas voltadas para aliviar a pobreza: o problema de focalização quando a renda não é diretamente observada”. In: XXII Encontro Brasileiro de Econometria, 2000, Campinas. Anais do XXII Encontro Brasileiro de Econometria. Rio de Janeiro: SBE.

BARROS, R. P., HENRIQUES, R., MENDONÇA, R. (2001) A estabilidade inaceitável: desigualdade e pobreza no Brasil. Texto para discussão, n. 800. Rio de Janeiro: IPEA - ISSN 1.415-4.765, jun.

COADY, A., GROSH, M., HODDINOTT, J. (2004) Targeting of Transfers in Developing Countries: Review of Lessons and Experience. [S. L.]: Banco Mundial/IFPRI.

CORNIA, G. A., STEWART, F. (1995) “Two Erros of Targeting”. In: D. van de Walle, K. Nead, Public spending and the poor: theory and evidence. Baltimore e Londres: Johns Hopkins University Press for the World Bank.

DE LA BRIERE, B., LINDERT, K. (2003) “Reforming Brazil's Cadastro Único to Improve the Targeting of the Bolsa Família Program”. DFID/World Bank. Out.

FARIA, A. L. C. (2006) "Aplicação do teste de elegibilidade multidimensional na definição do público-alvo beneficiário de políticas públicas". (Dissertação de mestrado em estudos populacionais e pesquisas sociais. ENCE/IBGE). Rio de Janeiro.

IBGE. (2003) “Microdados da pesquisa nacional por amostra de domicícilos PNAD”.

KERSTENETZKY, C. L. (2005) "O debate sobre políticas sociais: focalização ou universalização?” Trabalho apresentado no X Encontro Nacional da Sociedade de Economia Política, Instituto de Economia, Campinas, 24-27, maio.

LEGOVINI, A. (1999) “Targeting method for social programs”. Inter-American Development Bank, Poverty and Inequality Technical Notes, n. 1, Apr. 
MINISTÉRIO DA FAZENDA - SECRETARIA DE POLÍTICA ECONÔMICA (2003) “Gasto social do governo central: 2001 e 2002”. In: Revista Econômica, Rio de Janeiro, v. 5, n. 1, p. 9, jun.

NERI, M. (2003) "Focalização, universalização e transferências sociais". Revista Econômica, Rio de Janeiro, v. 5, n. 1, p. 163, jun.

PESSOA, D., SILVA, P. L.N. (1998) Análise de dados amostrais complexos. São Paulo: ABE, 187 p.

PINTO DE CARVALHO, A. (2005) “Decomposição o diferencial de salários no Brasil em 2003: uma aplicação dos procedimentos de Oaxaca e Heckman em pesquisas amostrais complexas”. Dissertação de Mestrado em Estudos Populacionais e Pesquisas Sociais. ENCE/ IBGE, Rio de Janeiro.

PREFEITURA DA CIDADE DE SÃO PAULO - SECRETARIA MUNICIPAL DO DESENVOLVIMENTO, TRABALHO E SOLIDARIEDADE (2003) "Desigualdade de renda e gastos sociais no Brasil: algumas evidências sobre o debate”. In: Revista Econômica, Rio de Janeiro, v. 5, n. 1, p. 71 , jun.

RELATÓRIO DE DESENVOLVIMENTO HUMANO DE 2004. PNUD. Disponível em < http://hdr. undp.org/reports/global/2004/ > Acesso em 09/03/2005.

ROCHA, S. (2003) Pobreza no Brasil: afinal, de que se trata?. Rio de Janeiro: FGV. 244 p.

SALM, C. (2003) "Privações sociais e gasto público". Revista Econômica, Rio de Janeiro, v. 5, n. 1, p. 83-90, jun.

SEN, A. (1995) “The Political Economy of Targeting”. In: D. van de Walle, K. Nead. Public spending and the poor: theory and evidence. Baltimore e Londres: Johns Hopkins University Press for the World Bank. 